\title{
Analysis of reconfigurable assembly system framing systems in automotive industry
}

\author{
Mohamad Zamri Md Zain ${ }^{1}$, Ahmad Majdi Abdul Rani ${ }^{2,}$, Mohd Amin Abd Majid ${ }^{1}$ \\ ${ }^{1}$ Mechanical Engineering Department, Universiti Teknologi PETRONAS, 31750 Tronoh, Perak, \\ Malaysia \\ ${ }^{2}$ Centre for Intelligent Signal \& Imaging Research (CISIR), Universiti Teknologi PETRONAS
}

\begin{abstract}
Current trend in automotive industry shows increasing demand for multiple models with lean production. Prior to that, automotive manufacturing systems evolved from mass production to flexible automation. Material handling systems and equipment in a single assembly line with multiple models require high investment but with low throughput thus making production cost relatively high. Current assembly process of side structure and undercarriage with downtime occurrence during assembly process affecting production performance (quality, cost and delivery). Manufacturing facilities should allow more flexibility and increase intelligence evolving toward novel reconfigurable assembly systems (RAS). RAS is envisaged capable of increasing factor flexibility and responsiveness by incorporating assembly jig, robot and framing, which could be next generation of world class automotive assembly systems. This project research proposes a new methodology of framework reconfigurable assembly systems principles in automotive framing systems i.e. enhance assembly process between side structure assembly and undercarriage assembly which a new RAS is capable to reconfigure the assembly processes of multiple model on a single assembly line. Simulation software (Witness) will be used to simulate and validate current and proposed assembly process. RAS is expected to be a solution for rapid change in structure and for a responsively adjustable production capacity. Quality, cost and delivery are production key parameters that can be achieved by implementing RAS.
\end{abstract}

\section{Introduction}

Current global economy scenario shows new enablers are needed in both product development and manufacturing systems due to open and unpredictable market changes. Automotive manufacturers explore economies of scale and scope through globally shared features of their products or models, for example: (1) sharing common automotive development, (2) collaborative engineering processes, (3) incorporated manufacturing processes. In small automotive industry, particularly in Association of Southeast Asian Nations (ASEAN) region; the primary issues with the current systems are capability to

* Corresponding author: mohzam11@yahoo.com 
produce multiple models in a single line and an integration between material handling and equipment. Thus to overcome these problems new trends evolution of automotive framing systems should be considered.

Based on Organisation Internationale des Constructuers d' Automobiles or The International Organization of Motor Vehicle Manufacturers (OICA), automotive manufacturer which Total Industry Volume (TIV) for passenger cars production annually less than 200,000 units can be categorized as small automotive industry [1]. Manufacturing enterprises are forced to re-evaluate their production paradigms, so that their products can be designed to maximize potential achievable variants, while the manufacturing systems to make them can be designed to operate efficiently by robustly accommodating future product changes, minimizing time to market and providing a reliable production base. Manufacturing facilities have to possess a high degree of flexibility, enabling mass customization of production. Mass customization is the capability of a firm to offer high productivity (product variety and overall volume), low manufacturing cost and also fast delivery [2].

Multiple models can now be assembled on a single production line. Normally, more than $70 \%$ of the tooling and equipment in a body shop are not specific to an individual model but can be used for all models produced. The production line can be planned to assemble various models, such as sedans, hatchbacks or SUVs (sport utility vehicles). While in a paint shop, all equipments are programmable to cover all styles efficiently and cost effectively. Also in the final assembly, most of major automakers are building more models, gained from global platforms, on the production lines that can simultaneously handle multiple products allowing for efficient utilization of people and equipment. Fig. 1 shows the automotive framing system assembling process through combination of a common under-body platform and side structure framing to become body in white (BIW). The automotive structure with the outer metal panels (skin) define the vehicle styling, which at the end become special appearance characteristics.

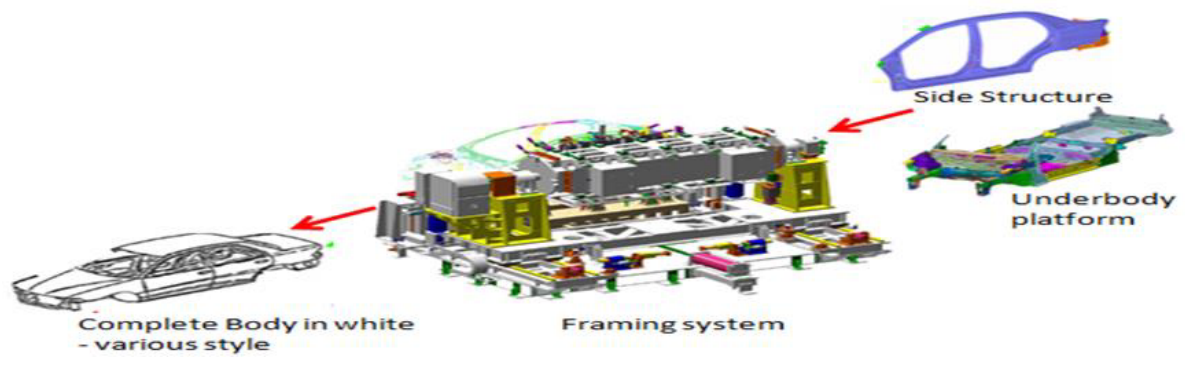

Fig. 1. Automotive framing system.

This paper focus on the manufacturing systems by identifying various potential flexible elements for product platform design and also identify their physical interaction with manufacturing mainbody framing systems. The hypothesis is that if the correct subsets of automotive body segments and production capabilities are designed considering for future flexibility based on the reconfigurability principles, then the manufacturing system can better adapt body styling changes, variants of family production without the need for tooling changeover with significant increase in throughputs.

\section{Optimization of assembly process}

Assembly includes all assembly or sub assembly processes and equipment which required to: 
a) Bring together, configure, orient, align and adjust components and materials to form the end product.

b) Physically attach parts, materials and components such as stapling, nailing, screwing, riveting, gluing, interlocking, wrapping, tying, fusing, welding, sewing, bonding, pegging, soldering, laminating, insertion, sealing, coupling and similar activities.

Assembly is an essential part of total manufacturing process which assembly cost typically $25 \%$ to $50 \%$ of total manufacturing cost. The total manufacturing cost consists of fixed cost and variable cost. When the costs are added, it has the following total cost in Eq. (1):

$$
T C=F C+V C(Q)
$$

where $T C=$ total annual cost $(\mathrm{RM} / \mathrm{yr}), F C=$ fixed annual cost $(\mathrm{RM} / \mathrm{yr}), V C=$ variable $\operatorname{cost}(\mathrm{RM} / \mathrm{pc})$, and $Q=$ annual quantity produced $(\mathrm{pc} / \mathrm{yr})$

Assembly system is a portion of manufacturing system, which carries out the assembly processes. Assembly systems can be classified in terms of different criteria. Based on system reconfigurability, assembly systems are classified into dedicated, flexible and reconfigurable. A dedicated manufacturing system is planned for manufacturing specific or customize product at a fixed line. While a flexible manufacturing system is designed for production of a part family, which consists of fixed hardware and fixed, but programmable, software to handle changes in work orders, production schedules, part programmes and tooling for several types of parts in the family. A reconfigurable assembly system (RAS) is designed at the outset for rapid change in structure, as well as in hardware and software components, in order to quickly adjust product capacity and functionality within a part family in response to sudden changes in market or in regulatory requirements.

It should be noted that the terminology 'reconfigurable' has many alternatives such as 'flexible', 'modular', 'agile', 'recomposable', 'retransformable', 'recomponentable' [4]; although these terminologies have a little different meanings, their creators have had the same motivation for them to deal with changes and uncertainties of the system. Among a challenge of current manufacturing systems is to deal with the changes and uncertainties in area of product functions, variations, product volume, delivery time and quality. RAS can be a solution to overcome the said challenges.

From academic perspective, flexibility as a metric of the system's behavior can be expressed in many forms, depending on the system's desired characteristics. So far several types of flexibility have been proposed such as product, operation, process, volume, expansion and labor flexibility [5]. G. Michalos et al. also highlighted that in general, four approaches could be distinguished in the design of an assembly system: (1) Manual assembly, (2) Fixed assembly, (3) Semi-automated assembly, (4) Flexible assembly

Platforms can be defined as a common infrastructure that serves as a backbone for multiple product or model variants. It cover common components, processes, and interfaces that allow end products to achieve unique variety in their product attributes by adding unique elements to product platforms. Unique elements are only found in individual variants, but not in the common platform [4]. A platform provides a common base for the assembling of a great number of vehicle variations, involving completely different models (e.g. Audi TT and Volkswagen Golf) and different model versions (i.e. 3 door, 5 door, coupe and sedan). Based on that, its required a factory to be flexible enough to handle this product variety. 


\section{Overview of automotive framing system}

'Framing system' is a process and the related basic structure for a precise positioning and securing under-body platform with the upper body components during assembling process. The body framing systems is focused because it is a critical stage in terms of assuring proper geometry and structural integrity of the automotive vehicle body. Nowadays, most passenger vehicles made comprises of 100-150 stamped metal panels, assembled into 1822 modules (sub-assemblies) in order to become a structural body. These modules are assembled into 3 systems to create the vehicle body formed (prior to painting, is referred to as body-in-white) in a multistep manufacturing process, during which the modules are joined together by welding. In order to assure the final body geometry according to design specifications, the panels to be welded are positioned (by locating pins and NC blocks), constrained by clamps and presented to the process by highly complex automated fixture devices.

Overall, a vehicle framing system can be divided into two subsystems, as shown in Fig. 2:

a) Under-body platform (consisting of up to 10-12 subassemblies).

b) BIW framing (3-5 sub-assemblies added prior to framing), grouped into up to 4 assembly cells.

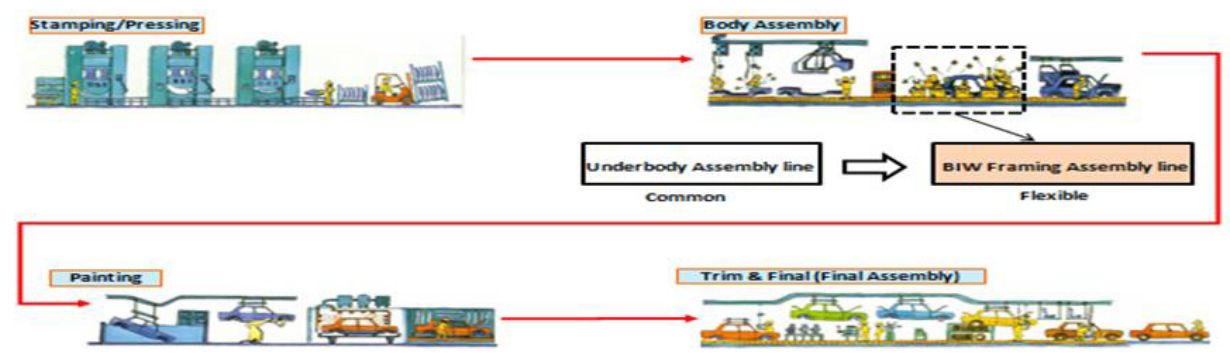

Fig. 2. Assembly process of BIW framing systems.

Main body jig or framing station has been dedicated and complex for a model of body. Over the years, different concepts of framing jigs have been developed with objective of high productivity and assured quality. However, with changing manufacturing strategy to attain lean inventory and more variety, flexibility of framing jig has become a more demanding requirement. A change-over or introduction small automotive of new models, manufacturing industry has three alternatives: (1) Change the old line with new one by stopping production for a considerable period, (2) Allocate the new line in different location, (3) Change manufacturing line for production of forthcoming models while production of the current model/s go on.

The best option for the manufacturers is the third alternative and flexibility is a necessity for reasons i.e. to improve mixed production, enormous investment for change over when the model is changed, stoppage of manufacturing lines prior to change over, surplus tied up production capacity during phasing out of model and huge manpower to change the layout and installation of new equipment [6].

Historically, all current framing systems have evolved from the Open-Gate and RoboGate system, initially developed in the 1980s. There are several types of framing systems i.e. (1) 2 slider framer, (2) Robot change framer, (3) Separated type robot framer, (4) Onebody type robot framer, (5) Manifold cylinder framer, (6) Roof robot framer, (7) Sliding framer system (Open-gate system), (8) Index framer 
Among the concern items with the current framing systems are currently the gates structure is integral and strongly coupled design; changeover times have to be accommodated for in the production plan, as the line has to stop running; gates storage systems require significant amount of floor space; overall high cost, high lead-times for engineering and build time; very expensive to manufacture and maintain; high cost of lost production due to downtime (breakdown or retooling) and high risk in capital investment.

\section{Evolution of automotive framing systems}

The aim in the future production systems is to quickly be able to adjust to product changes. A new trend come out in the manufacturing systems is called knowledge capture. Evolution/history of developments of vehicle framing systems are shown in Fig. 3. Due to markets increasingly require more customized products with shorter life cycles in the last few year, so manufacturing systems have evolved from mass production techniques through flexible automation and mass customization, to produce at mass production costs. Manufacturing facilities must incorporate more flexibility and intelligence, evolving toward reconfigurable assembly systems (RAS) prior to support effectiveness of manufacturing operations.

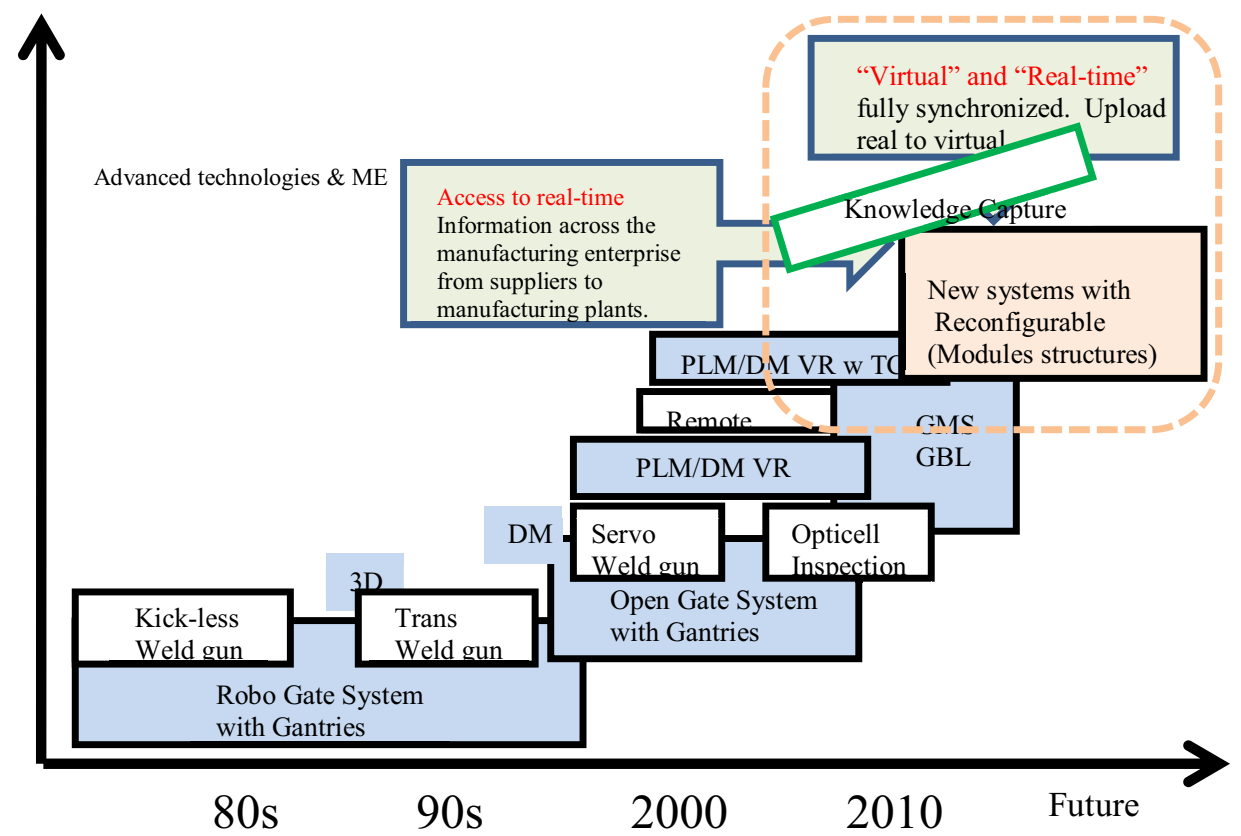

Fig. 3. Evolution of Automotive Framing Systems [4].

\section{Problem statement}

One of an element in the most of automotive manufacturer including small manufacturer to remain competitive is manufacturing cost. Therefore minimize manufacturing expenses always have been put priority in their operations. With limited flexibility that they have in order to achieve a good productivity i.e. increase of production volume, small car industry also have intention to produce multi model in their plant at current area. Sedan, Hatchback 
5-doors, Hatchback 3-doors, Coupe, MPV (Multi Purpose Van) and SUV (Sport Utility Vehicle) are among the models categorized under passenger car/vehicle.

Rigidity and complexity are characteristics of current production systems of BIW vehicle framing which it different with product structure. Experience, expertise and knowledge among the factors in automotive industry prior to enhance a systematic methodology because manufacturing process design and integration are subjective. The methodology can be a guideline for systems designers and developers in order to react quickly and more economically to unpredictable of market change by utilizing flexibility in both product and production systems. The hypothesis is a requirement to have a potential to assemble side structure and underbody at the single assembly line can be materialized if the right subset of vehicle body elements and assembly processes are designed with proper care for future flexibility, then the proposed production assembly system compatible with multiple models i.e. variants of family production.

\section{Research goal}

The goal is to reconfigure a production assembly process, to identify and incorporate flexibility in the assembly process of production, to verify the proposed reconfigurable assembly system through simulation and validation and respond to a requirement for implementing multiple model manufacturing system at Mainbody line and minimize production downtime.

\section{Research flow}

In this research, some kinds of methodology are being conducted as shown in the Fig. 4 below:

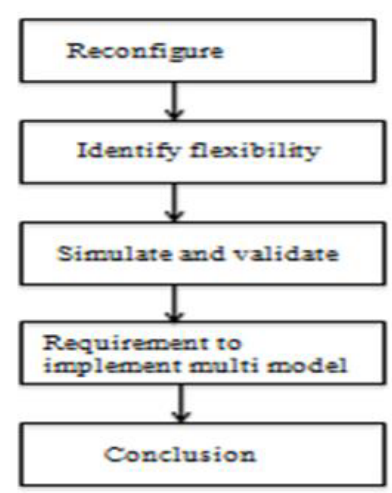

- Construct the study on Mainbody framing

systems through reconfiguration approach

- Incorporate flexibility in the assembly process of current production

- Verify proposed reconfigurable assembly system - Clarify variables and constraints

- Identify requirements to implement the framing systems on a single assembly line

- Reconfigure current assembly process at Mlainbody framing

Fig. 4. The research methodology flow.

In the methodology, simulation software is used i.e. WITNESS 12. Simulation model development is developed based on information from the automotive manufacturing plant data. 


\section{Results and discussion}

By changing Mainbody assembling process from Mainbody Presetting method to enhance Mainbody framing i.e. Robot gripper, some positive result are expected to achieve based on Quality, Cost and Delivery perspective. In term of quality, Mainbody accuracy percentage will be increased and become consistent. Cost of down time and loss of production can be reduced or eliminated and last but not least, delivery of production volume can be improved and achieve as per planned. Optimal number of models for a Mainbody framing also can be determined.

In manufacturing or assembling process, the results of product output are measured based on quality, cost and delivery. The manufacturer or assembler will enhance their current process and engineering know-how in order to achieve better result of quality, cost and delivery target indicators.

Body in White (BIW) is a jig for fitment of components parts in making an automotive CBU (Complete Build up Unit). Therefore, factor accuracy of the mainbody plays a major role in achieving good quality of the CBU. By achieving quality target accuracy of $85 \%$ for mainbody, it can help a lot to achieve quality of final product later. Gap, parts fitting and flushness can be improved through good accuracy of mainbody.

Operating in minimum cost is a requirement from the management. Profit is one of the key performance indicator of most of company at the end of financial year. Through reconfigurable assembly systems i.e. by changing assembly process from Mainbody Presetting to Mainbody Robot Gripper type, cost of downtime can be reduced. Cost of an opportunity loss also need to take in account once production output is not achieve target. For example, if daily production plan is to produce 400 units BIW but in actual only produce 300 units, meaning minus 100 units which is considered as opportunity loss.

Among the main key task in production is to deliver product or finish good as per delivery plan. Reconfigurable assembly systems can be a medium in order to achieve the delivery plan. Value analysis of mainbody sub systems can be validated through RAS approach.

\section{Conclusions}

Reconfigurable assembly systems (RAS) can be a solution for rapid change in automotive framing structure and for a responsively adjustable production capacity. It also contribute in improving manufacturing or production target indicators i.e. quality, cost and delivery. Mainbody accuracy percentage also be able to improve by implementing RAS. Future study also can be explored for Mainbody framing systems by using Aluminium material for side gate.

The author would like to be obliged to Ministry of Higher Education Malaysia (MOHE) for providing financial assistance.

\section{References}

1. des Constructeurs d'Automobiles, Organisation Internationale, World motor vehicle production by manufacturer (2012)

2. A. Al-Zaher, W. El Maraghy, Z.J Pasek, J. Mfg. Sys., 32, 436-448 (2013)

3. M.P. Groover, Automation, Production Systems and Computer-Integrated Manufacturing, 2nd Edition, Prentice Hall (2001)

4. Z.M. Bi, L. Wang, S.Y.T. Lang, Int. J. Mfg. Research, 2, 303-328 (2007)

5. G. Michalos, S. Makris, N. Papakostas, D. Mourtziz, G. Chryssolouris, CIRP J. Mfg. Sc. \& Tech. 2, 81- 91 (2010)

6. Drishtikona, Body Welding - Can it be flexible, http://www.drishtikona.com/books/automobile-manufacturing/ch6, 1-27 (2010) 